\title{
Isolated hemiataxia after supratentorial brain infarction
}

\author{
Gert-Jan Luijckx, Jelis Boiten, Jan Lodder, Lisette Heuts-van Raak, Jan Wilmink
}

\begin{abstract}
Acute isolated hemiataxia is in most cases due to infratentorial (cerebellar) stroke. It has only twice been described in supratentorial stroke-namely, after thalamic infarction and a capsular haemorrhage. Three patients with isolated hemiataxia after a supratentorial brain infarct are described. These patients were seen in a period of five years during which 899 patients with a first supratentorial brain infarct were registered. Clinically the hemiataxia was of the cerebellar type. In two patients, CT and MRI showed a small, deep (lacunar) infarct restricted to the posterior limb of the internal capsule, a site not previously reported in isolated hemiataxia. The third patient had a small, deep (lacunar) infarct in the thalamus extending into the adjacent posterior limb of the internal capsule. Isolated hemiataxia after a supratentorial brain infarct is a very rare clinical stroke syndrome. The cerebellar type hemiataxia was most likely caused by interruption of the cerebellar pathways at the level of the internal capsule. Our cases confirm prior observations that the cerebellar pathways run through the posterior part of the posterior limb of the internal capsule separately from the motor and sensory pathways.
\end{abstract}

\section{$(\Im$ Neurol Neurosurg Psychiatry 1994;57:742-744)}

Department of Neurology

G-J Luijckx

J Boiten

J Lodder

L H-v Raak

Department of Neuroradiology, University Hospital Maastricht, State University of

Limburg, Maastricht, The Netherlands J Wilmink

Correspondence to: Gert-Jan Luijckx, MD Department of Neurology, University Hospital Maastricht, PO Box 5800 $6200 \mathrm{AZ}$ Maastricht The Netherlands.

Received 19 October 1993. Accepted 8 November 1993
Hemiataxia after a supratentorial brain infarct is not uncommon. In most cases, however, hemiataxia is accompanied by either ipsilateral motor (ataxic hemiparesis) or sensory signs (hemiataxia-hypesthesia), or both (hypesthetic ataxic hemiparesis), whereas it rarely occurs in isolation. Isolated hemiataxia after supratentorial brain infarction has only once been described in thalamic infarction and after a small haemorrhage in the internal capsule. ${ }^{12}$ We now report on three patients with isolated hemiataxia after a supratentorial brain infarct. Two of these patients had a small, deep (lacunar) capsular infarct, a site not reported before in isolated hemiataxia.

Subjects and methods

The patients were selected from the
Maastricht Brain Infarct Registry, which is a prospective registry at the University Hospital of Maastricht of all patients with a first ever supratentorial brain infarct with symptoms lasting longer than 24 hours. This has been described in detail recently. ${ }^{34}$ All patients had either cranial CT, MRI, or both. Routine investigations included standard blood and urine tests, non-invasive carotid studies, and an ECG. Vascular risk factors, carotid stenosis, and cardioembolic sources were recorded. The patients were followed up prospectively every three to six months.

We selected those patients who presented with hemiataxia, defined as a unilateral incoordination on finger to finger, finger to nose and heel to shin tests with dysmetria, hypermetria, intention tremor, dysdiadochokinesia, or combinations of these signs.

\section{Results}

From July 1987 until February 1993, 899 patients with a first ever supratentorial brain infarct were registered. Of these, 47 patients (5.2\%; $95 \%$ confidence interval $(95 \% \mathrm{CI})$ $4 \cdot 9-5 \cdot 5 \%$ ) had hemiataxia, which was accompanied by either motor or sensory signs, or both, in 43 patients. Four of these 47 patients had isolated hemiataxia, in three of whom brain imaging showed a symptomatic lesion. Clinical features of these three patients together with CT and MRI findings are described in more detail in the following case reports:

\section{CASE 1}

A 63 year old man, with a history of hypertension and smoking suddenly developed clumsiness of the left arm and unsteadiness of gait without weakness. On admission the same day neurological examination showed left sided dysmetria, hypermetria, and intention tremor on finger to finger, finger to nose, and heel to shin tests. Somatosensory evoked potentials were normal.

On day 4 CT showed a hypodense lesion in the posterior limb of the right internal capsule, compatible with a small, deep (lacunar) infarct. After three months MRI showed the same lesion (figure; A,B). Neurological examination after six weeks was normal. Follow up at six months showed no abnormalities.

A 65 year old woman with a history of 

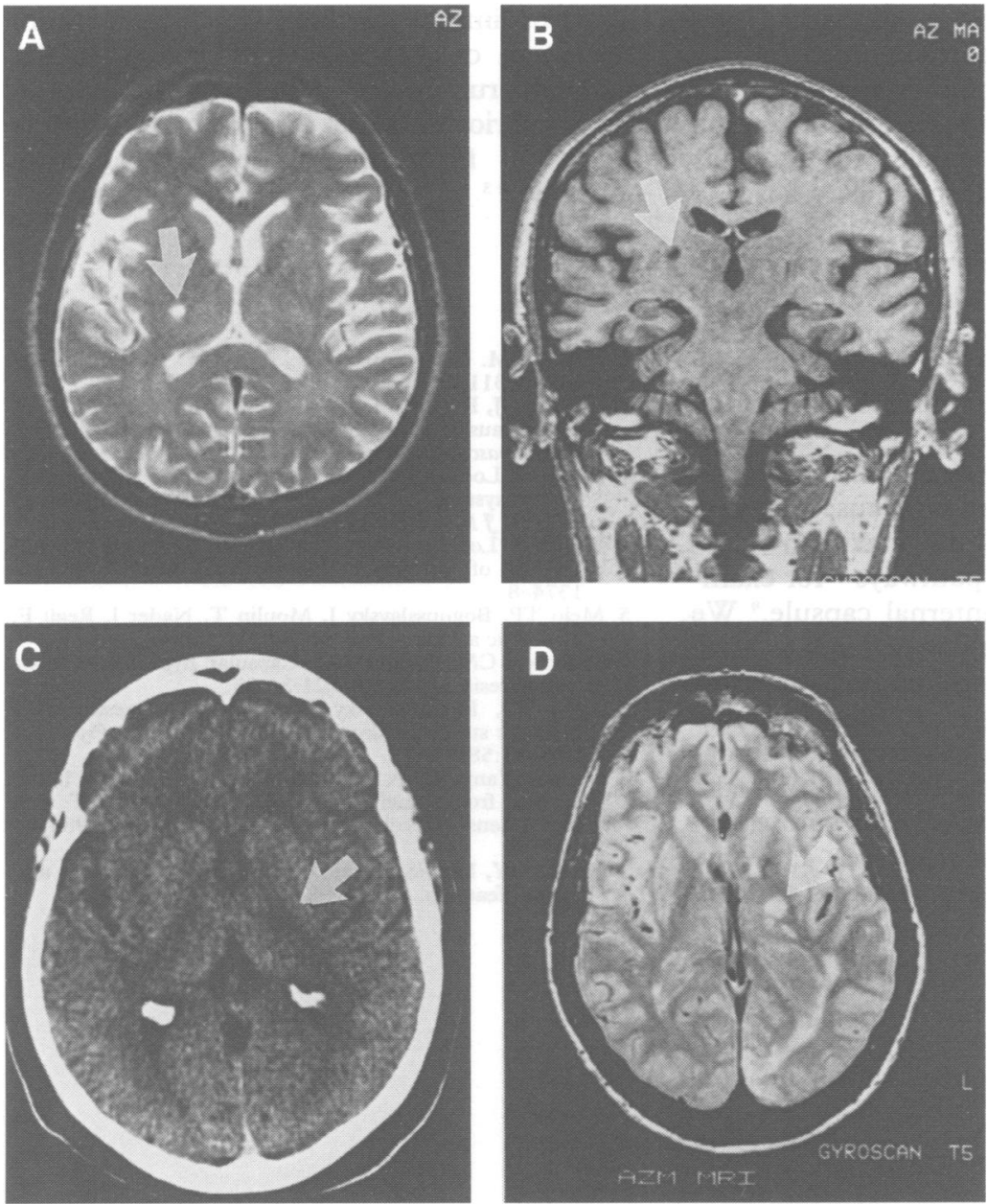

Figure (A) Transverse MRI, T2-weighted, of patient 1 showing a small deep lesion with high signal intensity in the posterior limb of the internal capsule on the right side (arrow): (B) Coronal T1-weighted MRI showing the same lesion in the posterior limb of the right internal capsule (arrow). The lesion does not involve the thalamus. (C) Transverse CT section of patient 2 showing a small hypodense ischaemic lesion in the posterior limb of the internal capsule on the left side (arrow). (D) Transverse proton density weighted MRI of patient 3 showing a lesion with high signal intensity in the left lateral thalamus extending into the adjacent posterior limb of the internal capsule (arrow). Also shown are two small adjacent satellite lesions. Note the cortical infarct in the territory of the left posterior cerebral artery that the patient had experienced four months after the initial infarct, just before this $M R I$ was made.

ischaemic heart disease and atrial fibrillation suddenly experienced clumsiness of the right hand with unsteadiness of gait without weakness. The symptoms had a stuttering onset, and were progressive during the first 48 hours. Neurological examination on day 3 showed right sided dysmetria, hypermetria, and intention tremor on coordination tests. Incoordination was more prominent in the arm than in the leg. On day 9 CT showed a small, hypodense lesion in the posterior limb of the left internal capsule compatible with a small, deep (lacunar) infarct (figure; C). Ataxia improved during the next three months. At three months the patient suddenly died of myocardial infarction.

CASE 3

A 68 year old man with a history of hypercholesterolaemia, complained of transient dysarthria, persisting sudden clumsiness of the right arm, and unsteadiness of gait without weakness. Neurological examination on the same day showed right sided dysmetria and hypermetria on finger to finger, finger to nose and heel to shin tests, and dysdiado- chokinesia. Ataxia of the arm was more severe than that of the leg. Somatosensory evoked potentials were normal. CT on day 8 showed a small, hypodense lesion in the left lateral thalamus extending into the adjacent posterior limb of the internal capsule compatible with a recent infarct. Ataxia improved during the next six weeks, at which time the neurological examination showed only slight ataxia on finger to finger and finger to nose tests. Four months later after the patient had had a recurrent cortical stroke in the same hemisphere, MRI showed the same thalamocapsular lesion (figure; D).

\section{Discussion}

Although acute isolated cerebellar ataxia is usually caused by cerebellar stroke our study shows that acute cerebellar hemiataxia may exceptionally be caused by a supratentorially located stroke. Our three patients with isolated hemiataxia and a symptomatic, supratentorially located ischemic brain lesion were seen in a period of five years during which we registered 899 patients in our brain infarct registry of all consecutive patients with a first supratentorial brain infarct. Obviously, isolated hemiataxia after a supratentorial stroke is a very rare clinical syndrome.

In previously reported series, hemiataxia after supratentorial brain lesions never occurred in isolation but was accompanied by either motor or sensory signs, or both. ${ }^{5-7}$ Isolated hemiataxia after supratentorial brain lesions has only been reported twice: Fisher described isolated hemiataxia after thalamic infarction, ${ }^{1}$ whereas we recently reported on isolated hemiataxia caused by a small capsular haemorrhage. ${ }^{2}$

Cases of ataxic hemiparesis, in which the cerebellar type ataxia is associated with ipsilateral motor weakness, are far more common than isolated hemiataxia cases. One could imagine that our patients also had hemiparesis at onset which had already disappeared at the time of examination. This seems unlikely, however, because, firstly, two of our patients were examined at the day of onset within a few hours; and secondly, in the third patient (case 2) the neurological symptoms were progressive during the first $\mathbf{4 8}$ hours after which the patient was examined showing only hemiataxia. In two of our patients ataxia was more prominent in the upper than in the lower limb, whereas in none of our patients was it more prominent in the lower limb. The hemiataxia was relatively mild with good resolution in all three cases.

Two of our patients had a small, deep (lacunar) infarct in the posterior limb of the internal capsule. Coronal and transverse MRI sections in one patient clearly showed that the lesion was restricted to the posterior limb of the internal capsule, whereas it did not involve the thalamus. Cases of isolated hemiataxia after a small, deep (lacunar) infarct restricted to the posterior limb of the internal capsule have not been reported before. Our third patient had an infarct of the lateral 
thalamus extending into the adjacent posterior limb of the internal capsule.

Hemiataxia in our patients was clinically of the cerebellar type. Disturbed proprioception as the cause of the ataxia was unlikely because there was no deep sensory loss on clinical testing, whereas eye closure did not worsen the ataxia. Moreover, the somatosensory evoked potentials, which were made in cases 1 and 3, were both normal. In ataxic hemiparesis the explanation for ataxia is still controversial. Some authors hypothesised that proprioceptive deficits-for example, resulting from damage to the ventral posterior nucleus of the thalamus-cause ataxia, ${ }^{8}$ whereas others ascribed the ataxia to interruption of the cerebellar pathways - for example, at the level of the internal capsule. ${ }^{9} \mathrm{We}$ considered hemiataxia in our patients to be of the cerebellar type. Therefore, it was most likely caused by damage to the cerebellar pathways, either the ascending dentatorubrothalamocortical pathway or the descending corticopontocerebellar pathway at the level of the internal capsule. The two patients with small, deep infarcts in the posterior limb of the internal capsule confirm our prior observations that the cerebellar pathways run through the posterior part of the posterior limb of the internal capsule separately from the motor and sensory pathways. ${ }^{23}$

1 Fisher CM. Lacunar infarcts. A review. Cerebrovasc Dis 1991;1:311-20.

2 Luijckx GJ, Boiten J, Lodder J, Wilmink J. Isolated hemiataxia caused by a small capsular hemorrhage [letter]. Cerebrovasc Dis 1993;3:381-2.

3 Boiten J, Lodder J. Discrete lesions in the sensorimotor control system. A clinical-topographical study of lacunar infarcts. F Neurol Sci 1991;105:150-4.

4 Boiten J, Lodder J. Lacunar infarcts: Pathogenesis and validity of the clinical syndromes. Stroke 1991;22: validity

5 Melo TP, Bogousslavsky J, Moulin T, Nader J, Regli F. Thalamic ataxia. $\mathcal{F}$ Neurol 1992;239:331-7.

6 Helgason CM, Wilbur AC. Capsular hypesthetic ataxic hemiparesis. Stroke 1990;21:24-33.

7 Melo TP, Bogousslavsky J. Hemiataxia-hypesthesia: a Melo TP, Bogousslavsky J. Hemiataxia-hypesthesia: a
thalamic stroke syndrome. I Neurol Neurosurg Psychiatry thalamic stroke

8 Mori E, Yamadori A, Kudo Y, Tabuchi M. Ataxic hemiparesis from small capsular hemorrhage. CT and somatosensory evoked potentials. Arch Neurol 1984;41: $1050-3$.

9 Huang CY, Lui FS. Ataxic hemiparesis, localizations and clinical features. Stroke 1984;14:363-6. 